CERN-TH-6341/91

HUTMP-91/B319

UTTG-32-91

\title{
A Generalized Construction of Mirror Manifolds
}

\author{
Per Berglund \\ CERN, Theory Division \\ CH-1211 Geneva 23, Switzerland \\ and \\ Theory Group, Department of Physics \\ University of Texas, Austin, TX 78712 \\ berglun@ cernvm.bitnet \\ Tristan Hübsch \\ Departments of Mathematics and Physics \\ Harvard University, Cambridge, MA 02138 \\ hubsch@ zariski.harvard.edu, @ huma1.bitnet
}

\begin{abstract}
We generalize the known method for explicit construction of mirror pairs of $(2,2)$-superconformal field theories, using the formalism of Landau-Ginzburg orbifolds. Geometrically, these theories are realized as Calabi-Yau hypersurfaces in weighted projective spaces. This generalization makes it possible to construct the mirror partners of many manifolds for which the mirror was not previously known.
\end{abstract}

CERN-TH-6341/91

$1 / 118$

- On leave from the Institute "Rudjer Bošković", Zagreb, Croatia. 


\section{Introduction}

String vacua which lead to $N=1$ space-time supersymmetry can be described by $(2,0)$-superconformal field theories [1]. Another approach is to consider Calabi-Yau manifolds as the classical background in which the string is propagating [2]. Although at first sight very different, it is by now well-known that a large class of Calabi-Yau spaces can be described in terms of $(2,2)$-superconformal field theories (see for example Refs. [3 3 -5]). In fact, it was conjectured that the Calabi-Yau spaces come in pairs, where for two spaces in such a pair the role of $(2,1)$-forms and $(1,1)$-forms respectively are interchanged ${ }^{1}$. Two such theories are said to form a mirror pair. Although the two respective underlying conformal field theories are isomorphic and differ only in the relative sign of the $U(1)$ currents in the $(2,2)$-superconformal field theory, it is far from straightforward to explicitly construct manifolds which exhibit the above mirror symmetry.

The first construction was given by Greene and Plesser [6], who considered the $3^{5}$ theory, i.e. a tensor product of five $A_{4}$ superconformal minimal models. The idea is to use the fact that [7]

$$
A_{k+1} / \mathbb{Z}_{k+2} \cong A_{k+1}
$$

the effect of the modding being a change of the relative sign of the left- and right-moving $U(1)$ charge. This procedure can be extended to tensor products of minimal models [6,8]. In particular,

$$
A_{4}^{5} / \mathbb{Z}_{5} \cong A_{4}^{5}
$$

Using the fact that a quintic hypersurface in $\mathbb{P}^{4}$, which we denote by $\mathbb{P}^{4}[5]$, can be thought of as the conformal field theory corresponding to $A_{4}^{5} / \mathbb{Z}_{5}$, where the quotient is the GSOtype projection, one finds that $\mathbb{P}^{4}[5] / \mathbb{Z}_{5}^{3}$ is the mirror to $\mathbb{P}^{4}[5]$. The class of theories which can be described by a polynomial (superpotential) of the Fermat type is however quite small. A larger class is formed by those polynomials which can be related to a Fermat type by a non-linear change of coordinates with a constant Jacobian [9, 10]. Hence the above construction for Fermat polynomials can also be used in this case to obtain the mirror manifold in a straightforward manner.

In this paper we describe a construction of the mirror theory from a $(2,2)$ superconformal field theory defined in terms of a Landau-Ginzburg field theory. Unlike in the constructions outlined above, we will not restrict (or even relate) the superpotential to the Fermat type polynomial, which corresponds to a tensor product of $A_{k}$-type minimal models. Instead, we consider the rather more general class of non-singular polynomials

1 We adopt the convention [1] where $(2,1)$-forms are equivalent to $U(1)$ charge- $(1,1)$ states in the conformal field theory language while $(1,1)$-forms are analogs of charge- $(-1,1)$ states. 
with the number of monomials the same as the number of coordinates. In order to find the mirror, we need to consider quotients of another theory whose defining polynomial is the transpose, in a sense that will be made precise, of the original one.

We will make use of the recently established ties [4,55,11] between the geometric point of view and the Landau-Ginzburg orbifold approach. Corresponding to a hypersurface $\mathcal{M}$ in a weighted projective space $\mathbb{P}_{\left(l_{1}, \ldots, l_{5}\right)}^{4}$ defined by $P\left(x_{i}\right)=0$, the $(2,2)$-superconformal field theory is determined by the superpotential $P\left(\Phi_{i}\right)$. The fact that $P\left(\Phi_{i}\right)$ is the same polynomial as $P\left(x_{i}\right)$ leads to some important identifications. In particular, the function ring of the variety $\mathcal{M}$ [12] and the chiral ring of the corresponding Landau-Ginzburg model [13] are identical. Moreover, the ring structure of the full $(p, q)$-cohomology on $\mathcal{M}$ can be identified in complete detail with the full ring of marginal operators of the LandauGinzburg orbifold-including untwisted and twisted, $(c, c)$ - and $(a, c)$-sectors [11]. Also, the scaling symmetry and the associated GSO-type projection correspond to the projectivity of the ambient space $\mathcal{M}$. This allows us to freely toggle between Calabi-Yau hypersurfaces and the corresponding Landau-Ginzburg orbifolds, and we make no notational distinction between them.

The paper is organized as follows. We first give the general construction in Section 2. In Section 3, we work out an explicit example and present the general arguments and explicit computations in verification of the mirror pairing of $\mathcal{W}$ with $\mathcal{M}$. Section 4 contains our closing remarks, and some technical details are left for the Appendices.

\section{The Construction}

Consider a smooth hypersurface $\mathcal{M}$ in a weighted projective space $\mathbb{P}_{\left(l_{1}, \ldots, l_{5}\right)}^{4}$ of dimension four. The generalization to other dimensions is straightforward ${ }^{2}$. For $\mathcal{M}$ to be Calabi-Yau, it must be defined as the zero-set of a polynomial of degree $d=\sum_{i=1}^{5} l_{i}$ :

$$
P\left(x_{1}, \ldots, x_{5}\right)=0, \quad\left(x_{1}, \ldots, x_{5}\right) \in \mathbb{P}_{\left(l_{1}, \ldots, l_{5}\right)}^{4},
$$

and $x_{i}$ has scaling weight $l_{i}$. The corresponding statement for a $(2,2)$-superconformal field theory leads to a theory with central charge $c=9$. Let us also define $Q_{\mathcal{M}}=\mathbb{Z}_{d}$ to be the scaling symmetry $Q_{\mathcal{M}}$ (requiring $\lambda^{d}=1$ ),

$$
x_{i} \mapsto \lambda^{l_{i}} x_{i}, \quad P\left(x_{i}\right) \mapsto \lambda^{d} P\left(x_{i}\right)
$$

${ }^{2} n>4$ is relevant when considering theories with more than five fields, such as complete intersection Calabi-Yau manifolds and tensor products of models from the ADE series. 
associated with $P$. The associated Landau-Ginzburg orbifold is obtained from the LandauGinzburg field theory with superpotential $P$, by implementing the $\mathbb{Z}_{d}$ GSO-type projection. This Landau-Ginzburg orbifold will also be denoted by $\mathcal{M}$ and we note that this $\mathbb{Z}_{d}$ becomes the so-called 'quantum symmetry' of $\mathcal{M}$ [14]. The group of all phase symmetries of $P$, excluding $Q_{\mathcal{M}}$, is called the 'geometric symmetry'3 of $\mathcal{M}$ and is denoted $G_{\mathcal{M}}$.

For the general analysis of Refs. [4,5,11] to apply, we must require the system of gradients $\partial_{i} P$ to vanish only at the origin $x_{i}=0$. In this note, we also restrict $P\left(x_{i}\right)$ to be a sum of only as many monomials as there are coordinates (five in the present case), which is clearly the minimal choice. Under this minimality condition it is straightforward to extend the analysis of Chapter 13 in Ref. [15] and we list all 16 minimal non-singular polynomials in Table 1; they are contained in the list obtained previously in Ref. [16]. Of course, by allowing more than the minimal number of monomials, more general superpotentials are obtained and a similar study of such a larger class is under way; we hope to report on these results in a detailed study.

Given a model $\mathcal{M}$ with one of the superpotentials from Table 1, we now want to find the mirror model $\mathcal{W}$. The idea is to construct another model, $\mathcal{W}$, such that the roles of the quantum symmetry and the geometrical symmetry are interchanged [10], that is,

$$
\begin{aligned}
Q_{\mathcal{M}} \cong G_{\mathcal{W}} \\
G_{\mathcal{M}} \cong Q_{\mathcal{W}} .
\end{aligned}
$$

Note that $\mathcal{W}$ will in general be a quotient of a manifold with the fixed points blown up.

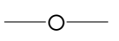

Let us now study the different cases at hand. For the first (Fermat-type) polynomial in Table $1, \mathcal{W}$ is obtained by dividing $\mathcal{M}$ by the action of $\Pi_{\mathcal{M}}$, the group of phase symmetries which leave the $(3,0)$-form $\Omega$ invariant. This is the technique used by Greene and Plesser as mentioned previously [6,17].

We turn therefore to those polynomials for which dividing by the action of $\Pi_{\mathcal{M}}$ does not yield the mirror. To demonstrate the procedure, let us describe it in detail for one of the polynomials in Table 1. The other cases will then follow easily (see also Section 3 and Tables 1 and 2).

From Table 1, we take for example

$$
P=x_{1}^{a_{1}} x_{2}+x_{2}^{a_{2}} x_{3}+x_{3}^{a_{3}} x_{4}+x_{4}^{a_{4}} x_{5}+x_{5}^{a_{5}} .
$$

3 The full geometric symmetry group will of course also contain permutation symmetries, but we include these separately as usual. 
To this superpotential, we may associate the matrix of exponents

$$
P \simeq\left[\begin{array}{ccccc}
a_{1} & 0 & 0 & 0 & 0 \\
1 & a_{2} & 0 & 0 & 0 \\
0 & 1 & a_{3} & 0 & 0 \\
0 & 0 & 1 & a_{4} & 0 \\
0 & 0 & 0 & 1 & a_{5}
\end{array}\right]
$$

whose columns are the degree vectors of the respective monomials of $P$. It is convenient at this point to note that $P$ has a $\mathbb{Z}_{a_{1} \cdots a_{5}}$ phase symmetry. To see this, let the charge of $x_{5}$ be $\vartheta_{5}=1 / a_{5}$. For the monomial $x_{4}^{a_{4}} x_{5}$ to transform with an integral (not necessarily unity!) charge, we may choose $\vartheta_{4}=\frac{-1}{a_{4} a_{5}}$. Thereafter, $\vartheta_{3}=\frac{+1}{a_{3} a_{4} a_{5}}, \vartheta_{2}=\frac{-1}{a_{2} a_{3} a_{4} a_{5}}, \vartheta_{1}=\frac{+1}{a_{1} a_{2} a_{3} a_{4} a_{5}}$ and we have a manifest $\mathbb{Z}_{a_{1} \cdots a_{5}}$ action.

The new polynomial $\widehat{P}$ is then defined to correspond to the transposed matrix and so is

$$
\widehat{P}=x_{5}^{a_{5}} x_{4}+x_{4}^{a_{4}} x_{3}+x_{3}^{a_{3}} x_{2}+x_{2}^{a_{2}} x_{1}+x_{1}^{a_{1}} \quad, \quad \widehat{P} \simeq\left[\begin{array}{ccccc}
a_{1} & 1 & 0 & 0 & 0 \\
0 & a_{2} & 1 & 0 & 0 \\
0 & 0 & a_{3} & 1 & 0 \\
0 & 0 & 0 & a_{4} & 1 \\
0 & 0 & 0 & 0 & a_{5}
\end{array}\right] .
$$

We say that $\widehat{P}$ is the transpose ${ }^{4}$ of $P$. Of course, transposing again gives back $P$. Apart from this transposition, $\widehat{P}$ still belongs to the same class of polynomials as $P$ (see Table 1 ). Most crucially, the total phase symmetry of $\widehat{P}$ is again $\mathbb{Z}_{a_{1} \cdots a_{5}}$, i.e. $Q_{\mathcal{M}} \times G_{\mathcal{M}}$.

The zero locus of the transposed polynomial $\widehat{P}$ defines a hypersurface $\widehat{\mathcal{W}}=$ $\mathbb{P}_{\left(\hat{l}_{1}, \ldots, \hat{l}_{5}\right)}^{4}[\widehat{d}]$, of which the degree $\widehat{d}$ and the weights $\widehat{l_{i}}$ are determined by $\widehat{P}$ using $(2.2)$. In general, Eqs. (2.3) will not be fulfilled because $Q_{\widehat{\mathcal{W}}} \subset Q_{\mathcal{W}}$, but is typically smaller than $Q_{\mathcal{W}}$, see Section 3 and Table 2 for examples. To enlarge $Q_{\widehat{\mathcal{W}}}$, we must divide $\widehat{\mathcal{W}}$ by a suitable group of phase symmetries $H$ such that

$$
\begin{aligned}
& Q_{\mathcal{W}} \stackrel{\text { def }}{=} Q_{\widehat{\mathcal{W}}} \times H \cong G_{\mathcal{M}}, \\
& G_{\mathcal{W}} \stackrel{\text { def }}{=} Q_{\widehat{\mathcal{W}}} / H \cong Q_{\mathcal{M}} .
\end{aligned}
$$

We then assert that $\mathcal{W}=\widehat{\mathcal{W}} / H$ is the mirror of $\mathcal{M}$ and also check, case by case, that a suitable $H$ does indeed exist. In Table 1 , we list the transpose $\widehat{P}$ for each of the defining polynomials (superpotentials) $P$. We are not aware of a definition of $H$ in closed form and suitable for display with the general classes as in Table 1; see Table 2 for some examples.

We note in passing that all Fermat-type polynomials are self-transposed. From the point of view of our construction, this is precisely the reason why the method of Greene

4 It appears that this transposition is related to the $\left(\mathbb{Z}_{2}\right)$ reversal in the relative sign between the $U(1)$-currents in the underlying $(2,2)$-superconformal field theory. 
and Plesser works for the Fermat polynomials. More generally, if a polynomial $P$ is selftransposed, whether of the Fermat type or not ${ }^{5}$, we find that the mirror of $\mathcal{M}$ is a quotient of $P$ such that Eqs. (2.3) are fulfilled. This accounts for the original class of mirror pairs [6, 17].

With regard to the ADE classification of the $N=2$ superconformal minimal models, it is worth pointing out the following interesting fact. It is easy to check that all models except $D_{k}(k>3)$ are invariant under transposition. In particular, the $E_{7}$ polynomial $\left(x^{3}+x y^{3}\right)$ is also self-transposed. Since it occurs in the $1 \cdot 16^{3}$ model, this explains why the mirror of the Schimmrigk manifold [18], $\mathcal{M}$, is just $\mathcal{M} / \Pi_{\mathcal{M}}$.

For $D_{k}$ we have the following defining polynomial:

$$
P\left(D_{k}\right)=x_{1}^{k-1}+x_{1} x_{2}^{2}
$$

the transpose of which is

$$
\widehat{P}\left(D_{k}\right)=x_{2}^{2}+x_{2} x_{1}^{k-1}
$$

For $k>3$ we see that $P \neq \widehat{P}$, and so the mirror cannot in general be found by the standard technique. That is, explicit calculation of the $(c, c)$ - and $(a, c)$-rings of relevant operators shows that unless $k$ is even no quotient of $P\left(D_{k}\right)$ can be identified with the mirror of $P\left(D_{k}\right)$. Instead, there is a $\widehat{P}\left(D_{k}\right) / \mathbb{Z}_{2(k-1)}$ Landau-Ginzburg orbifold which may be identified with the mirror of $P\left(D_{k}\right)$ (the details are presented in Appendix A). 6

Given our short discussion of the $P\left(D_{k}\right)$ and $\widehat{P}\left(D_{k}\right) / \mathbb{Z}_{2(k-1)}$ Landau-Ginzburg models and the details in Appendix A, it is easy to see that the basic argument for the mirror pairing of $\mathcal{W}$ with $\mathcal{M}$ is the same as in the usual case [6]. There, the mirror map followed from two facts. Firstly,

$$
P\left(A_{k}\right) / \mathbb{Z}_{k} \approx P\left(A_{k}\right)
$$

for a 1-variable $A$-type Landau-Ginzburg model. Second, such models could be combined into a $c=9$ theory, where the isomorphism (2.10) reverses the sign of the Euler characteristic. Likewise here, we break up the polynomials in Table 1 into irreducible $n$-variable models $(n=2, \ldots, 5)$ and generalizing the above $P\left(D_{k}\right) \approx \widehat{P}\left(D_{k}\right) / \mathbb{Z}_{2(k-1)}$ paradigm, we find that the transposition and quotient by a suitable $H$ provide the generalization of the

5 There exist special self-transposed examples such as Eq. (2.4), with $l_{i}=l_{5-i}$ and so $a_{i}=a_{5-i}$. Most often however, transposition associates weighted hypersurfaces of very different weights.

6 Note that if we think of the $D_{k}$ model as a $\mathbb{Z}_{2}$ orbifold of $A_{2 k-3}$ and a trivial quadratic piece the mirror of $D_{k}$ is then given in a straightforward manner as a $\mathbb{Z}_{2(k-1)}$ orbifold of $A_{2 k-3}+A_{1}$. 
isomorphism (2.10) for all the irreducible $n$-variable models used in Table 1. The application of this to the "compound" polynomials in Table 1-and so also the mirror pairing of $\mathcal{W}$ with $\mathcal{M}$-is then straightforward.

Greene and Plesser recently described a somewhat larger class of models [10]. They considered mirror pairs $(\mathcal{M}, \mathcal{W})$, where $\mathcal{W}$ is constructed by first performing a fractional but holomorphic change of coordinates to get to a Fermat polynomial. They then deform the latter so as to obtain $\mathcal{W}$, with Eqs. (2.3) satisfied.

Although it is always possible to get from one of the polynomials in Table 1 to a Fermat polynomial, by a fractional holomorphic coordinate transformation, in general it will not be possible to also deform to a $\mathcal{W}$ such that the roles of the quantum and geometric symmetry are interchanged. Thus, the construction described in this paper generalizes the known method for finding the mirror manifold and makes the $\mathbb{Z}_{2}$ transposition manifest.

\section{An Example}

To illustrate the method let us consider the following example. The manifold below is listed in Ref. [16] as one without a mirror. Let $\mathcal{M}$ be a degree-75 hypersurface, $\mathcal{M}=$ $\mathbb{P}_{(5,8,12,15,35)}^{4}[75]_{+6}^{27,30}$, with $\chi_{E}=+6, b_{2,1}=27$, and $b_{1,1}=30$, defined by the zero locus of

$$
P=x_{1}^{15}+x_{2}^{5} x_{5}+x_{1} x_{5}^{2}+x_{3}^{5} x_{4}+x_{4}^{5}
$$

Note that $Q_{\mathcal{M}}=\mathbb{Z}_{75}$ and $G_{\mathcal{M}}=\mathbb{Z}_{25} \times \mathbb{Z}_{2}$. To $P$ we can associate the following matrix (see Eq. (2.5))

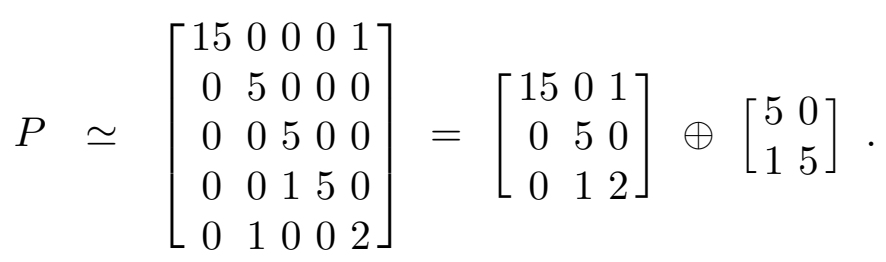

Next, we construct

$$
\widehat{P}=x_{1}^{15} x_{5}+x_{5}^{2} x_{2}+x_{2}^{5}+x_{4}^{5} x_{3}+x_{3}^{5},
$$

corresponding to the transposed matrix. Using Eq. (2.4), we find that $\widehat{P}=0$ defines a hypersurface $\widehat{\mathcal{W}}=\mathbb{P}_{(1,5,5,4,10)}^{4}[25]_{-120}^{69,9}$. Note that $Q_{\widehat{\mathcal{W}}}=\mathbb{Z}_{25}$ and $G_{\widehat{\mathcal{W}}}=\mathbb{Z}_{2} \times \mathbb{Z}_{75}$. Thus $Q_{\widehat{\mathcal{W}}}$ is not isomorphic to $G_{\mathcal{M}}$. However, on dividing by ${ }^{7}$

$$
H \stackrel{\text { def }}{=}\left(\mathbb{Z}_{2}: 1,0,0,0,1\right),
$$

7 We will use the notation $\left(\mathbb{Z}_{k}: r_{1}, r_{2}, r_{3}, r_{4}, r_{5}\right)$ for a $\mathbb{Z}_{k}$ symmetry with the action $\left(x_{1}, x_{2}, x_{3}, x_{4}, x_{5}\right) \rightarrow\left(\alpha^{r_{1}} x_{1}, \alpha^{r_{2}} x_{2}, \alpha^{r_{3}} x_{3}, \alpha^{r_{4}} x_{4}, \alpha^{r_{5}} x_{5}\right)$, where $\alpha^{k}=1$. 
we find that $\mathcal{W}=\widehat{\mathcal{W}} / H$ satisfies Eqs. (2.3) and identify $\mathcal{W}$ as the mirror of $\mathcal{M}$.

Using the method described in Appendix B, it is now straightforward to find not only $\chi_{E}$ but also $b_{2,1}$ and $b_{1,1}$. For the example in Section 3, we find $\chi_{E}(\mathcal{W})=-6$ and that $b_{2,1}=30$ and $b_{1,1}=27$. Hence, as expected from the general arguments above, $\mathcal{W}$ satisfies the basic numerical requirements for the mirror of $\mathcal{M}$.

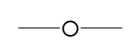

One may wonder if there exists a polynomial whose zero locus in a weighted projective space is the manifold $\mathcal{W}$ as constructed in the above example. After all, taking the quotient by $H$ may be enforced by the following fractional change of coordinates:

$$
\left(x_{1}, x_{2}, x_{3}, x_{4}, x_{5}\right)=\left(\tilde{x}_{1}^{1 / 2}, \tilde{x}_{2}, \tilde{x}_{3}, \tilde{x}_{4}, \tilde{x}_{5} \tilde{x}_{1}^{1 / 2}\right) .
$$

This gives us the polynomial

$$
\widetilde{P}=\tilde{x}_{1}^{8} \tilde{x}_{5}+\tilde{x}_{5}^{2} \tilde{x}_{1} \tilde{x}_{2}+\tilde{x}_{2}^{5}+\tilde{x}_{4}^{5} \tilde{x}_{3}+\tilde{x}_{3}^{5} .
$$

It is easy to check that $\widetilde{P}=0$ describes a degree-25 hypersurface in $\widetilde{\mathcal{W}}=\mathbb{P}_{(2,5,5,4,9)}^{4}$. However, $\widetilde{P}$ is not transverse at $p^{\sharp}=(0,0,0,0,1)$. Hence we cannot expect Vafa's formula for the Euler number [24] (see also Eq. (B.1)) to give us the correct spectrum as it only holds for smooth manifolds, that is non-degenerate Landau-Ginzburg orbifolds. Note however that $p^{\sharp}$ is in the fixed point set of $H$ and is also a local $\mathbb{Z}_{9}$-singularity as inherited from the weighted projective space. Interpreting (as usual) the Landau-Ginzburg orbifold as a blow-up at the fixed point set, the singularity may actually be smoothed completely and the situation may not be as bad as it seems. In a local coordinate system with $\tilde{x}_{5}=1$ and $u=\left(\tilde{x}_{4}^{5}+\tilde{x}_{3}^{4}\right)$ at $p^{\sharp}$, we have

$$
\left.\widetilde{P}\right|_{p^{\sharp}}=\tilde{x}_{1}^{8}+\tilde{x}_{1} \tilde{x}_{2}+\tilde{x}_{2}^{5}+u \tilde{x}_{3},\left.\quad \operatorname{det}\left[\partial^{2} \widetilde{P}\right]\right|_{p^{\sharp}}=1 .
$$

The singularity is therefore also a node, the Landau-Ginzburg orbifold is degenerate and we are unable to compute the numerical characteristics reliably [19].

In spite of the technical problems in computing the correct spectrum for a nontransverse polynomial, we believe that $\widetilde{\mathcal{W}}$ indeed is the mirror to $\mathcal{M}$. We base this conjecture on the fact that an appropriate resolution of the singular $\widetilde{\mathcal{W}}$ may be identified with the quotient $\widehat{\mathcal{W}} / H$. Note, however, that $\widetilde{\mathcal{W}}$ and $\mathcal{W}$ will correspond in general to two different points in the moduli space. This would lead to a special class of Calabi-Yau conifolds [20], which are non-transverse precisely at the fixed points of the scaling symmetry $Q_{\widetilde{\mathcal{W}}}$. It would be interesting to list all non-transverse polynomials of the above type to see if they would complete the list of weighted projective spaces [16] - as far as providing every manifold there with its mirror.

In Table 2, we give several more examples of theories for which the mirror was not previously known. As well as being of potential phenomenological interest, we hope that they will illustrate the ease with which the mirror model is constructed. 


\section{Discussion and Conclusions}

The cautious reader may worry whether there is a hidden caveat to our argument for the mirror pairing of $\mathcal{W}$ with $\mathcal{M}$. Indeed, the only completely unambiguous proof would have us compute the (correctly) normalized three-point functions $\left\langle\phi_{(1,1)}^{3}\right\rangle$ and $\left\langle\phi_{(-1,1)}^{3}\right\rangle$ in the underlying $(2,2)$-superconformal field theory. If

$$
\left\langle\phi_{(1,1)}^{3}\right\rangle_{\mathcal{M}}=\left\langle\phi_{(-1,1)}^{3}\right\rangle_{\mathcal{W}}, \quad\left\langle\phi_{(1,-1)}^{3}\right\rangle_{\mathcal{M}}=\left\langle\phi_{(1,1)}^{3}\right\rangle_{\mathcal{W}}
$$

the proof is complete, since the three-point functions completely determine the $(2,2)$ superconformal field theory.

Unfortunately, it does not seem possible at this time to complete the above calculation, because the $(2,2)$-superconformal field theories we find are not tensor products of minimal models and the correct normalizations seem to elude us. Nevertheless, using the ring structure of the Landau-Ginzburg orbifolds, specified through the Jacobian ideal generated by the system of gradients of the superpotential, as well as the quantum and geometric symmetries, it is straightforward to show that the general structure of the Yukawa couplings, such as the zeros, is the same.

Without delving into the calculation (which will be presented in full detail elsewhere [21]), for the example discussed earlier we find a one-to-one relation between the $(1,1)$ states in the superconformal field theory corresponding to $\mathcal{M}$ and the $(-1,1)$ states in $\mathcal{W}$ and vice versa.

We have described a technique which generalizes the existing methods of obtaining the mirror manifold to a given hypersurface in a weighted projective space. The method also applies for complete intersection Calabi-Yau manifolds, for which the covering space can be embedded in a (product of) weighted projective space(s) - as long as the superpotential has as many monomials as there are variables. We conjecture that in general the mirror $\mathcal{W}$ to a manifold $\mathcal{M}$ does not have to be described by a transverse polynomial-although $\mathcal{W}$ can be expressed as a quotient of a non-singular covering space $\widehat{\mathcal{W}}$ and the non-transversality occurs at fixed points of $Q_{\mathcal{W}}$. The straightforward way in which the method is applied makes us believe that one ought to be able to mechanize the procedure in terms of a computer code. From the list in Ref. [16], one would then be able to obtain a very large class of mirror pairs. Although there may still remain manifolds without a constructed mirror, we shall be a step closer to verifying that every Calabi-Yau manifold indeed has a mirror.

Acknowledgements: P.B. acknowledges useful discussions with P. Candelas , E. Derrick, X. de la Ossa and J. Louis. P.B. was supported by the Foundation BlanceflorBoncompagni-Ludovisi née Bildt, the Fulbright Program, a University of Texas Fellowship, 
and in part by the NSF grant PHY 9009850 and the Robert A. Welch foundation. T.H. was supported by the DOE grant DE-FG02-88ER-25065 and would also like to thank the Department of Mathematics of the National Tsing-Hua University at Hsinchu, Taiwan, for the warm hospitality during the time when part of this research was completed. 


\section{Appendix A. The Mirror of $D_{k}$}

In this appendix, we will show that the mirror to $D_{k}$ is given by $\widehat{P}\left(D_{k}\right) / \mathbb{Z}_{2(k-1)}$ for $k>2$. We have that the defining polynomial for $D_{k}$ is given by

$$
P\left(D_{k}\right)=x^{k-1}+x y^{2} .
$$

The charges are $q_{x}=\frac{1}{k-1}$ and $q_{y}=\frac{k-2}{2(k-1)}$. The corresponding Landau-Ginzburg theory has the following ring structure

$$
\begin{aligned}
(c, c): & \left\{|0\rangle_{(c, c)}^{(0)}, x|0\rangle_{(c, c)}^{(0)}, \ldots, x^{k-2}|0\rangle_{(c, c)}^{(0)}, y|0\rangle_{(c, c)}^{(0)}\right\}_{k} \\
(a, c): & \left\{|0\rangle_{(a, c)}^{(0)}\right\}_{1}
\end{aligned}
$$

where the subscripts $k$ and 1 indicate the number of states in the $(c, c)$ and $(a, c)$ rings respectively. To find the mirror theory we consider quotients of $D_{k}$. Depending on whether $k$ is even or odd, there are two situations.

For $k$ even, the scaling symmetry $j$ of $D_{k}$ is $\left(\mathbb{Z}_{k-1}: 1, \frac{k-2}{2}\right)$. Using the result in Ref. [22] for the transformation of a given state under a symmetry, we find the states which are invariant under $j$. This gives rise to the ring structure

$$
\begin{aligned}
(c, c): & \left\{|0\rangle_{(c, c)}^{(0)}\right\}_{1} \\
(a, c): & \left\{|0\rangle_{(a, c)}^{(0)},|0\rangle_{(a, c)}^{(k-2)}, \ldots,|0\rangle_{(a, c)}^{(2)}, x^{\frac{k}{2}-1}|0\rangle_{(a, c)}^{(1)}, y|0\rangle_{(a, c)}^{(1)}\right\}_{k}
\end{aligned}
$$

It is easy to check, using Eq. (B.4), that the $(a, c)$ states above have the same charges as the $(c, c)$ states in the $D_{k}$ theory except that $q_{L} \rightarrow-q_{L}$. So $D_{k} / \mathbb{Z}_{k-1}$ is the mirror to $D_{k}$ for $k$ even.

When $k$ is odd the scaling symmetry $j$ is $\left(\mathbb{Z}_{2(k-1)}: 2, k-2\right)$. A calculation similar to the one above shows that, unless $k=3, D_{k} / j$ is not the mirror; neither is any other quotient. We then turn to the transposed polynomial for $D_{k}$,

$$
\widehat{P}\left(D_{k}\right)=y^{2}+y x^{k-1}
$$

which has charges $q_{1}=\frac{1}{2(k-1)}$ and $q_{2}=\frac{1}{2}$. Note that for all $k$ (even and odd), the scaling symmetry is $\widehat{\jmath}=\left(\mathbb{Z}_{2(k-1)}: 1, k-1\right)$. It is then straightforward to obtain the ring structure for the LG-orbifold $\widehat{P}\left(D_{k}\right) / \widehat{\jmath}$ :

$$
\begin{aligned}
(c, c): & \left\{|0\rangle_{(c, c)}^{(0)}\right\}_{1} \\
(a, c): & \left\{|0\rangle_{(a, c)}^{(0)},|0\rangle_{(a, c)}^{(2(k-1)-2)}, \ldots,|0\rangle_{(a, c)}^{(2)}, y|0\rangle_{(a, c)}^{(1)}\right\}_{k} .
\end{aligned}
$$

Note that for $\ell=2 p+1$, the states $x^{k-2}|0\rangle_{(a, c)}^{(1)}$ are not projected out, but $\left.\partial_{x} P\left(D_{k}\right)\right|_{y=0}=$ $x^{k-2}$ and the state is in the ideal. (We restrict to $y=0$ because $\Theta_{y}(2 p+1) \notin \mathbb{Z}$.) Using Eq. (2.3), one can check that the charges are the same as for the $(c, c)$ states in the $D_{k}$ model, with a change of sign for $q_{L}$. Thus, $\widehat{P}\left(D_{k}\right) / \widehat{\jmath}$ is the mirror of $D_{k}$ for any $k>2$. 


\section{Appendix B. Computation of $\chi_{E}, b_{2,1}$ and $b_{1,1}$}

We next turn to compute the $\chi_{E}, b_{2,1}$ and $b_{1,1}$ for $\mathcal{W}$ (see also Ref. [23]). To this end, we need the expression for the Euler number for a weighted projective hypersurface [24,25] :

$$
\chi_{E}=\frac{1}{d} \sum_{r, \ell=0}^{d-1}(-1)^{(D-N)(r+\ell+r \ell)} \prod_{\ell q_{i}, r q_{i} \in \mathbb{Z}}\left(1-\frac{1}{q_{i}}\right),
$$

where $D=3$ is the dimension of the Calabi-Yau space, $N=5$ is the number of homogeneous coordinates, and $q_{i}=\ell_{i} / d$. We now want to generalize Eq. (B.1) so that it can also be valid for a quotient. First, rewrite it as

$$
\chi_{E}=\sum_{r=0}^{d-1} S_{r}
$$

where

$$
S_{r}=\frac{1}{d} \sum_{\ell=0}^{d-1}(-1)^{(D-N)(r+\ell+r \ell)} \prod_{\Theta_{i}(\ell), \Theta_{i}(r) \in \mathbb{Z}}\left(1-\frac{1}{q_{i}}\right) .
$$

Note that $r$ and $\ell$ run over all twisted sectors, including the sectors due to dividing by the discrete symmetry; $\Theta_{i}(\ell)$ is the $i^{t h}$ twist charge from the $\ell^{\text {th }}$ twisted sector and similarly for $\Theta_{i}(r)$. This is a generalization of $\ell q_{i}$ and $r q_{i}$ in Eq. (B.1) [22,11]. The point of writing $\chi_{E}$ in the form (B.2) is that it resembles the usual expression for the Euler number. In fact, the $S_{r}$ will determine the Hodge numbers.

To extract $b_{2,1}$ and $b_{1,1}$, we need to know which sectors contribute to $(2,1)$ - and $(1,1)$-forms, respectively. The simplest way to see this is by looking at the conformal field theory. Since $\chi_{E}$ is given by Eq. (B.1), it is enough to consider $b_{2,1}$. Recall that we associate charge- $(1,1)$ states to $(2,1)$-forms. The question is then which sectors contain $(1,1)$ states. To answer this, we need the expression for the charges of the Ramond vacuum in the $\ell^{t h}$ twisted sector [24]:

$$
J_{0}|0\rangle_{R}^{(\ell)}=\left\{ \pm\left[\sum_{\Theta_{i}(\ell) \notin \mathbb{Z}}\left(\Theta_{i}(\ell)-\left[\Theta_{i}(\ell)\right]-\frac{1}{2}\right)\right]+\left[\sum_{\Theta_{i}(\ell) \in \mathbb{Z}}\left(q_{i}-\frac{1}{2}\right)\right]\right\}|0\rangle_{R}^{(\ell)} .
$$

The $|0\rangle_{(c, c)}^{(\ell)}$ vacua are obtained by spectral flow $\mathcal{U}_{(1 / 2,1 / 2)}$, of charges $\left(\frac{c}{6}, \frac{c}{6}\right)$. It is then an easy exercise to find those sectors that contain marginal states of charge $(1,1)$. So we find that

$$
\begin{aligned}
& b_{2,1}=\frac{1}{2} b_{3}-1=-\frac{1}{2} \sum_{r_{+}} S_{r_{+}}-1 \\
& b_{1,1}=\frac{1}{2} \chi_{E}-b_{2,1}=\frac{1}{2} \sum_{r \neq r_{+}} S_{r}+1
\end{aligned}
$$


where $r_{+}$runs over the sectors contributing charge- $(1,1)$ states. The reason for the form of the expression for $b_{2,1}$ is that $S_{r_{+}}$also contains an equal number of $(2,2)$ states as well as one $(0,0)$ and one $(3,3)$ state respectively from the untwisted sector, $p=0$. The analogy between 3 -forms and integral $(c, c)$-states is self-evident and well-known. 


\begin{tabular}{|c|c|}
\hline$P$ & $\widehat{P}$ \\
\hline$x_{1}^{a_{1}}+x_{2}^{a_{2}}+x_{3}^{a_{3}}+x_{4}^{a_{4}}+x_{5}^{a_{5}}$ & $x_{1}^{a_{1}}+x_{2}^{a_{2}}+x_{3}^{a_{3}}+x_{4}^{a_{4}}+x_{5}^{a_{5}}$ \\
\hline$x_{1}^{a_{1}} x_{2}+x_{2}^{a_{2}}+x_{3}^{a_{3}}+x_{4}^{a_{4}}+x_{5}^{a_{5}}$ & $x_{2}^{a_{2}} x_{1}+x_{1}^{a_{1}}+x_{3}^{a_{3}}+x_{4}^{a_{4}}+x_{5}^{a_{5}}$ \\
\hline$x_{1}^{a_{1}} x_{2}+x_{2}^{a_{2}} x_{3}+x_{3}^{a_{3}}+x_{4}^{a_{4}}+x_{5}^{a_{5}}$ & $x_{3}^{a_{3}} x_{2}+x_{2}^{a_{2}} x_{1}+x_{1}^{a_{1}}+x_{4}^{a_{4}}+x_{5}^{a_{5}}$ \\
\hline$x_{1}^{a_{1}} x_{2}+x_{2}^{a_{2}} x_{3}+x_{3}^{a_{3}} x_{4}+x_{4}^{a_{4}}+x_{5}^{a_{5}}$ & $x_{4}^{a_{4}} x_{3}+x_{3}^{a_{3}} x_{2}+x_{2}^{a_{2}} x_{1}+x_{1}^{a_{1}}+x_{5}^{a_{5}}$ \\
\hline$x_{1}^{a_{1}} x_{2}+x_{2}^{a_{2}} x_{3}+x_{3}^{a_{3}} x_{4}+x_{4}^{a_{4}} x_{5}+x_{5}^{a_{5}}$ & $x_{5}^{a_{5}} x_{4}+x_{4}^{a_{4}} x_{3}+x_{3}^{a_{3}} x_{2}+x_{2}^{a_{2}} x_{1}+x_{1}^{a_{1}}$ \\
\hline$x_{1}^{a_{1}} x_{2}+x_{2}^{a_{2}}+x_{3}^{a_{3}} x_{4}+x_{4}^{a_{4}}+x_{5}^{a_{5}}$ & $x_{2}^{a_{2}} x_{1}+x_{1}^{a_{1}}+x_{4}^{a_{4}} x_{4}+x_{3}^{a_{3}}+x_{5}^{a_{5}}$ \\
\hline$x_{1}^{a_{1}} x_{2}+x_{2}^{a_{2}} x_{3}+x_{3}^{a_{3}}+x_{4}^{a_{4}} x_{5}+x_{5}^{a_{5}}$ & $x_{3}^{a_{3}} x_{2}+x_{2}^{a_{2}} x_{1}+x_{1}^{a_{1}}+x_{5}^{a_{5}}+x_{5} x_{4}^{a_{4}}$ \\
\hline$x_{1}^{a_{1}}+x_{2}^{a_{2}}+x_{3}^{a_{3}}+x_{4}^{a_{4}} x_{5}+x_{5}^{a_{5}} x_{4}$ & $x_{1}^{a_{1}}+x_{2}^{a_{2}}+x_{3}^{a_{3}}+x_{4}^{a_{4}} x_{5}+x_{5}^{a_{5}} x_{4}$ \\
\hline$x_{1}^{a_{1}}+x_{2}^{a_{2}} x_{3}+x_{3}^{a_{3}}+x_{4}^{a_{4}} x_{5}+x_{5}^{a_{5}} x_{4}$ & $x_{1}^{a_{1}}+x_{3}^{a_{3}} x_{2}+x_{2}^{a_{2}}+x_{4}^{a_{4}} x_{5}+x_{5}^{a_{5}} x_{4}$ \\
\hline$x_{1}^{a_{1}} x_{2}+x_{2}^{a_{2}} x_{3}+x_{3}^{a_{3}}+x_{4}^{a_{4}} x_{5}+x_{5}^{a_{5}} x_{4}$ & $x_{3}^{a_{3}} x_{2}+x_{2}^{a_{2}} x_{1}+x_{1}^{a_{1}}+x_{4}^{a_{4}} x_{5}+x_{5}^{a_{5}} x_{4}$ \\
\hline$x_{1}^{a_{1}}+x_{2}^{a_{2}} x_{3}+x_{3}^{a_{3}} x_{2}+x_{4}^{a_{4}} x_{5}+x_{5}^{a_{5}} x_{4}$ & $x_{1}^{a_{1}}+x_{2}^{a_{2}} x_{3}+x_{3}^{a_{3}} x_{2}+x_{4}^{a_{4}} x_{5}+x_{5}^{a_{5}} x_{4}$ \\
\hline$x_{1}^{a_{1}}+x_{2}^{a_{2}}+x_{3}^{a_{3}} x_{4}+x_{4}^{a_{4}} x_{5}+x_{5}^{a_{5}} x_{3}$ & $x_{1}^{a_{1}}+x_{2}^{a_{2}}+x_{5}^{a_{5}} x_{4}+x_{4}^{a_{4}} x_{3}+x_{3}^{a_{3}} x_{5}$ \\
\hline$x_{1}^{a_{1}} x_{2}+x_{2}^{a_{2}}+x_{3}^{a_{3}} x_{4}+x_{4}^{a_{4}} x_{5}+x_{5}^{a_{5}} x_{3}$ & $x_{2}^{a_{2}} x_{1}+x_{1}^{a_{1}}+x_{5}^{a_{5}} x_{4}+x_{4}^{a_{4}} x_{3}+x_{3}^{a_{3}} x_{5}$ \\
\hline$x_{1}^{a_{1}} x_{2}+x_{2}^{a_{2}} x_{1}+x_{3}^{a_{3}} x_{4}+x_{4}^{a_{4}} x_{5}+x_{5}^{a_{5}} x_{3}$ & $x_{1}^{a_{1}} x_{2}+x_{2}^{a_{2}} x_{1}+x_{5}^{a_{5}} x_{4}+x_{4}^{a_{4}} x_{3}+x_{3}^{a_{3}} x_{5}$ \\
\hline$x_{1}^{a_{1}}+x_{2}^{a_{2}} x_{3}+x_{3}^{a_{3}} x_{4}+x_{4}^{a_{4}} x_{5}+x_{5}^{a_{5}} x_{2}$ & $x_{1}^{a_{1}}+x_{5}^{a_{5}} x_{4}+x_{4}^{a_{4}} x_{3}+x_{3}^{a_{3}} x_{2}+x_{2}^{a_{2}} x_{5}$ \\
\hline$x_{1}^{a_{1}} x_{2}+x_{2}^{a_{2}} x_{3}+x_{3}^{a_{3}} x_{4}+x_{4}^{a_{4}} x_{5}+x_{5}^{a_{5}} x_{1}$ & $x_{5}^{a_{5}} x_{4}+x_{4}^{a_{4}} x_{3}+x_{3}^{a_{3}} x_{2}+x_{2}^{a_{2}} x_{1}+x_{1}^{a_{1}} x_{5}$ \\
\hline
\end{tabular}

Table 1: $P=0$ defines a hypersurface in a weighted projective 4 -space. $\widehat{P}=0$ gives the covering space, $\widehat{\mathcal{W}}$, of $\mathcal{W}$, the mirror of $\mathcal{M}$. $\mathcal{W}$ will in general be a quotient of $\widehat{\mathcal{W}}$. 


\begin{tabular}{|c|c|c|}
\hline $\mathcal{M}, \widehat{\mathcal{W}}$ & $P, \widehat{P}$ & $\mathcal{W}$ \\
\hline $\mathbb{P}_{(2,8,29,49,59)}^{4}[147]_{-6}^{51,48}$ & $x_{2}^{11} x_{5}+x_{5}^{2} x_{3}+x_{3}^{5} x_{1}+x_{1}^{49} x_{4}+x_{4}^{3}$ & \\
\hline $\mathbb{P}_{(1,5,6,18,25)}^{4}[55]_{-138}^{97,28}$ & $x_{4}^{3} x_{1}+x_{1}^{49} x_{3}+x_{3}^{5} x_{5}+x_{5}^{2} x_{2}+x_{2}^{11}$ & $\widehat{\mathcal{W}} /\left(\mathbb{Z}_{2}: 1,0,1,1,1\right)$ \\
\hline $\mathbb{P}_{(3,4,14,21,21)}^{4}[63]_{-6}^{35,32}$ & $x_{2}^{15} x_{1}+x_{1}^{21}+x_{3}^{3} x_{4}+x_{4}^{3}+x_{5}^{3}$ & \\
\hline $\mathbb{P}_{(2,3,10,15,15)}^{4}[45]_{-54}^{49,22}$ & $x_{1}^{21} x_{2}+x_{2}^{15}+x_{4}^{3} x_{3}+x_{3}^{3}+x_{5}^{3}$ & $\widehat{\mathcal{W}} /\left(\mathbb{Z}_{3}: 1,0,1,1,0\right)$ \\
\hline $\mathbb{P}_{(3,5,8,24,35)}^{4}[75]_{-6}^{43,40}$ & $x_{1}^{25}+x_{2}^{15}+x_{3}^{5} x_{5}+x_{4}^{3} x_{1}+x_{5}^{2} x_{2}$ & \\
\hline $\mathbb{P}_{(2,3,15,25,30)}^{4}[75]_{-96}^{75,27}$ & $x_{1}^{25} x_{4}+x_{4}^{3}+x_{2}^{15} x_{5}+x_{5}^{2} x_{3}+x_{3}^{5}$ & $\widehat{\mathcal{W}} /\left(\mathbb{Z}_{2}: 0,1,0,0,1\right)$ \\
\hline $\mathbb{P}_{(4,5,26,65,95)}^{4}[195]_{+6}^{67,70}$ & $x_{1}^{25} x_{5}+x_{5}^{2} x_{2}+x_{2}^{39}+x_{3}^{5} x_{4}+x_{4}^{3}$ & \\
\hline $\mathbb{P}_{(1,3,15,20,36)}^{4}[75]_{-228}^{145,31}$ & $x_{2}^{39} x_{5}+x_{5}^{2} x_{1}+x_{1}^{25}+x_{4}^{3} x_{3}+x_{3}^{5}$ & $\widehat{\mathcal{W}} /\left(\mathbb{Z}_{2}: 1,0,0,0,1\right)$ \\
\hline $\mathbb{P}_{(5,6,14,45,65)}^{4}[135]_{+6}^{42,45}$ & $x_{3}^{5} x_{5}+x_{5}^{2} x_{1}+x_{1}^{27}+x_{2}^{15} x_{4}+x_{4}^{3}$ & \\
\hline $\mathbb{P}_{(1,3,9,14,18)}^{4}[45]_{-144}^{95,23}$ & $x_{1}^{27} x_{5}+x_{5}^{2} x_{3}+x_{3}^{5}+x_{4}^{3} x_{2}+x_{2}^{15}$ & $\widehat{\mathcal{W}} /\left(\mathbb{Z}_{2}: 1,0,0,0,1\right)$ \\
\hline $\mathbb{P}_{(5,8,12,15,35)}^{4}[75]_{+6}^{27,30}$ & $x_{2}^{5} x_{5}+x_{5}^{2} x_{1}+x_{1}^{15}+x_{3}^{5} x_{4}+x_{4}^{5}$ & \\
\hline $\mathbb{P}_{(1,5,5,4,10)}^{4}[25]_{-120}^{69,9}$ & $x_{1}^{15} x_{5}+x_{5}^{2} x_{2}+x_{2}^{5}+x_{4}^{5} x_{3}+x_{3}^{5}$ & $\widehat{\mathcal{W}} /\left(\mathbb{Z}_{2}: 1,0,0,0,1\right)$ \\
\hline $\mathbb{P}_{(2,6,9,17,17)}^{4}[51]_{+6}^{31,34}$ & $x_{4}^{3}+x_{1}^{17} x_{5}+x_{5}^{3}+x_{2}^{7} x_{3}+x_{3}^{5} x_{2}$ & \\
\hline $\mathbb{P}_{(3,6,9,17,16)}^{4}[51]_{-102}^{66,51}$ & $x_{4}^{3}+x_{5}^{3} x_{1}+x_{1}^{17}+x_{3}^{5} x_{2}+x_{2}^{7} x_{3}$ & $\widehat{\mathcal{W}} /\left(\mathbb{Z}_{2}: 0,1,1,0,0\right)$ \\
\hline $\mathbb{P}_{(4,4,11,17,19)}^{4}[55]_{-6}^{24,21}$ & $x_{1}^{11} x_{3}+x_{3}^{5}+x_{2}^{9} x_{5}+x_{5}^{2} x_{4}+x_{4}^{3} x_{2}$ & \\
\hline $\mathbb{P}_{(1,1,2,2,5)}^{4}[11]_{-210}^{109,4}$ & $x_{3}^{5} x_{1}+x_{1}^{11}+x_{4}^{3} x_{5}+x_{5}^{2} x_{2}+x_{2}^{9} x_{4}$ & $\widehat{\mathcal{W}} /\left(\mathbb{Z}_{5}: 0,1,1,1,2\right)$ \\
\hline
\end{tabular}

Table 2: A list of $\mathcal{M}$ and their mirrors $\mathcal{W}$. $\widehat{\mathcal{W}}$ is defined by $\widehat{P}=0$. A GSO-type $\mathbb{Z}_{d^{-}}$ projection is implicitly understood, where $d$ is the degree of the defining polynomial. $\mathcal{M}$ are manifolds for which no mirror was listed in Ref. [16]. 


\section{References}

[1] For a review and references, see L. Dixon: in Superstrings, Unified Theories and Cosmology 1987, eds. G. Furlan et al. (World Scientific, Singapore, 1988) p. 67-127.

[2] P. Candelas, G. Horowitz, A. Strominger and E. Witten: Nucl. Phys. B258 (1985) 46.

[3] D. Gepner: Phys. Lett.199B (1987)380, "String theory on Calabi-Yau manifolds: the three generations case", Princeton University report (December 1987, unpublished).

[4] B.R. Greene, C. Vafa and N.P. Warner: Nucl.Phys. B324 (1989) 371;

J.I. Latorre and C.A. Lütken: Phys. Lett.222B (1989) 55;

S.J. Gates and T. Hübsch: Phys. Lett. 226 (1989) 100, Nucl.Phys. B343 (1990) 741;

B.R. Greene: Commun. Math. Phys. 130 (1990) 335.

[5] S. Cecotti, L. Girardello and A. Pasquinucci: Nucl. Phys.B328 (1989) 701, Int.J. Mod.Phys. A6 (1991) 2427;

S. Cecotti: Int. J. Mod. Phys. A6 (1991) 1749, Nucl. Phys. B355 (1991) 755.

[6] B.R. Greene and M.R. Plesser: Nucl. Phys. B338 (1990) 15.

[7] D. Gepner and Z. Qiu: Nucl. Phys. B285 (1987) 423.

[8] D. Gepner: Nucl. Phys. B296 (1988) 757.

[9] M. Lynker and R. Schimmrigk: Phys. Lett. 249B (1990) 237;

R. Schimmrigk: Mirror Symmetry in String Theory and Fractional Transformations, to appear in the proceedings of the PASCOS-91 Symposium, Boston 1991

[10] B.R. Greene and M.R. Plesser: Cornell and Yale University preprints CLNS 91-1109, YCTP-P32-91.

[11] P. Berglund, B. Greene and T. Hübsch: "Classical vs. Quantum Geometry of Compactification", University of Texas report UTTG-21-91 (1991).

[12] P. Griffiths and J. Harris: Principles of Algebraic Geometry (John Wiley, New York, 1978).

[13] W. Lerche, C. Vafa and N. Warner: Nucl. Phys. B324 (1989) 427.

[14] C. Vafa: Mod. Phys. Lett. A4 (1989) 1615.

[15] V.I. Arnold, S.M. Gusein-Zade and A.N. Varchenko: Singularities of Differentiable Maps, Vol. I (Birkhäuser, Boston, 1985).

[16] P. Candelas, M. Lynker and R. Schimmrigk: Nucl. Phys. B341 (1990) 383.

[17] P.S. Aspinwall, C.A. Lütken and G.G. Ross: Phys. Lett. 241B (1990) 373.

[18] R. Schimmrigk: Phys. Lett.193B (1987) 175.

[19] T. Hübsch: Mod. Phys. Lett. A6 (1991) 207, Class. Quant. Grav. 8 (1991) L31.

[20] P. Candelas, P.S. Green and T. Hübsch: Nucl. Phys. B330 (1990) 49.

[21] P. Berglund and T. Hübsch: in preparation.

[22] K. Intrilligator and C. Vafa: Nucl. Phys. B339 (1990) 95.

[23] S.-S. Roan: Int. J. Math. 2 (1991) 439.

[24] C. Vafa: Mod. Phys. Lett. A4 (1989) 1169.

[25] S.-S. Roan: Int. J. Math. 1 (1990) 211. 